Citation: M. Muscarà, A. Romano (2020) Didattica e apprendimento nei musei nell'era della pandemia di COVID-19. Media Education 11(2): 61-73. doi: 10.36253/me-9645

Received: August, 2020

Accepted: September, 2020

Published: December, 2020

Copyright: @2020 M. Muscarà,A. Romano. This is an open access, peerreviewed article published by Firenze University Press (http://www.fupress. $\mathrm{com} / \mathrm{me}$ ) and distributed under the terms of the Creative Commons Attribution License, which permits unrestricted use, distribution, and reproduction in any medium, provided the original author and source are credited.

Data Availability Statement: All relevant data are within the paper and its Supporting Information files.

Competing Interests: The Author(s) declare(s) no conflict of interest.

\section{Didattica e apprendimento nei musei nell'era della pandemia di COVID-19 1}

\section{Teaching and learning in museums in the era of the COVID-19 pandemic}

\author{
Marinella Muscarà ${ }^{\star}$, Alessandro Romano \\ Università Kore di Enna \\ *Correspondig author. E-mail: marinella.muscara@unikore.it; alessandro.romano20@ \\ unikorestudent.it
}

Sommario. Partendo dalla considerazione che il museo non rappresenta esclusivamente un luogo di conservazione, collezione e promozione del patrimonio culturale ma un vitale spazio di apprendimento, l'articolo indaga il ruolo che le tecnologie e i media hanno svolto durante la prima fase di diffusione della pandemia di Covid-19 in ambito museale. In particolare, gli autori riflettono sulle questioni del riconoscimento di alcune pratiche museali come pratiche didattiche, sull'impiego delle tecnologie per l'educazione al patrimonio culturale e per la sua fruizione e sulle iniziative digitali proposte dai musei per riallacciare la relazione con i pubblici durante il periodo di isolamento sociale. Si delinea pertanto la necessità, anche nei contesti di apprendimento non formale, di riconsiderare la funzione dei media per la valorizzazione delle pratiche d'insegnamento-apprendimento nei musei delineando nuove prospettive ed implicazioni didattiche.

Parole chiave: didattica museale, ICT, patrimonio culturale, Covid-19.

\begin{abstract}
Moving from the consideration that the museum does not represent only a place of preservation, collection and promotion of cultural heritage but a vital learning space, the article aims to investigate the role that technologies and media have played in the museum during the first phase of the spread of the Covid-19 pandemic. In particular, the authors reflect on the issues of the recognition of some museum practices as educational practices, on the use of technologies for cultural heritage education and for its use and on the digital initiatives proposed by museums in order to reconnect with the variety publics, in particular children and young people, during the period of social isolation. There is therefore a need, also in the context of non-formal learning, to reconsider the value of ICT to redesign and reshape teaching and learning practices, outlining new perspectives and educational implications.
\end{abstract}

Key words: museum education, ICT, cultural heritage, Covid-19. 


\section{INTRODUZIONE}

L'emergenza sanitaria che ha interessato il nostro Paese a tutti i livelli ha costretto le agenzie educative formali e non formali ad adottare modalità straordinarie di riorganizzazione delle attività didattiche, allo scopo di garantire a tutti il diritto all'educazione e alla formazione. Anche le istituzioni museali e i luoghi della cultura sono stati investiti dalla necessità di ripensare e di riallacciare la trama della relazione educativa interrotta bruscamente durante il periodo del lockdown con la molteplicità dei pubblici. Il patrimonio culturale, nelle sue componenti tangibili e intangibili, costituisce infatti un potente fattore chiave di sviluppo sociale ed economico in quanto risorsa preziosissima nei settori dell'istruzione, dell'occupazione, del turismo e dello sviluppo sostenibile (cfr. Consiglio d'Europa, 2017). Il valore del patrimonio culturale non risiede esclusivamente nella funzione di testimone della cultura di un gruppo umano ma esso si esplica anche nella capacità che ha di garantire lo sviluppo tanto dell'intera società quanto del singolo individuo. Anche per tale potenzialità, che si genera dall'esperienza con il cultural heritage, la Convenzione ONU sui diritti dell'infanzia e dell'adolescenza stabilisce che: «States Parties shall respect and promote the right of the child to participate fully in cultural and artistic life and shall encourage the provision of appropriate and equal opportunities for cultural, artistic, recreational and leisure activity»(United Nations, 1989, art. 31).

Il raggiungimento di tali obiettivi di sviluppo individuale e collettivo può avvenire, indica chiaramente il Consiglio d'Europa con la Recommendation of the Committee of Ministers to member States on the European Cultural Heritage Strategy for the 21st century del 2017, attraverso l'adozione di politiche statali volte alla promozione di azioni di educazione al patrimonio culturale tanto negli adulti quanto nei bambini. Se, infatti, il Consiglio d'Europa già nel 1998 aveva sottolineato l'importanza dell'educazione al patrimonio intesa come «a teaching approach based on cultural heritage, incorporating active educational methods, cross-curricular approaches, a partnership between the fields of education and culture and employing the widest variety of modes of communication and expression" (Consiglio d'Europa, 1998, p. 31), con la Raccomandazione del 2017 si conferma in ambito europeo l'importanza della dimensione dell'apprendimento legata al patrimonio culturale. A tale riguardo, non sorprende che una delle tre componenti ${ }^{2}$ fondamentali dell'intera Strategia Europea per il Patrimonio Culturale del $21^{\circ}$ secolo sia costituita proprio dal 'knowledge and education' che "focuses on the relationship between heritage and shared knowledge, covering awareness raising, training and research» (Consiglio d'Europa, 2017, p. 42).

Tra gli attori sociali direttamente coinvolti nella relazione tra patrimonio e conoscenza condivisa, oltre naturalmente alla scuola e alle università, i musei e i luoghi della cultura costituiscono soggetti affidabili proprio per la loro capacità di intercettare i bisogni del territorio e di contribuire all'elaborazione di visioni sociali su fenomeni complessi che coinvolgono l'intera società. I musei quindi, attraverso l'esercizio della loro funzione di presidi territoriali per l'educazione al patrimonio culturale e per lo sviluppo di competenze trasversali, operano come agenzie educative particolari in cui prendono forma fenomeni di apprendimento informale, non formale e talvolta anche formale (cfr. Crowley et al., 2014). L'osservazione delle pratiche museali e il loro riconoscimento come pratiche educative a tutti gli effetti sembrano potere richiamare la didattica e la pedagogia a un maggiore azione responsiva e responsabile nel settore della formazione al patrimonio culturale, contribuendo anche a spiegare e interpretare le relazioni emergenti tra tecnologia, mediatori digitali e strategie per la fruizione e l'educazione del cultural heritage. In misura sempre maggiore, infatti, i musei ricorrono oggi alle potenzialità offerte dall'utilizzo degli strumenti tecnologici e digitali per sviluppare nuove relazioni tra i diversi pubblici, operando un potenziamento delle abilità percettive del visitatore e delle capacità narrative degli oggetti della collezione. Sebbene, anche in ambito museale, non si sia ancora realizzato pienamente l'auspicato processo di innovazione digitale, l'isolamento sociale imposto dai provvedimenti per il controllo e contenimento della diffusione della pandemia da Covid-19 ha obbligato le istituzioni museali a ricorrere ai mediatori digitali, al web e ai social network per garantire la continuità dell'azione educativa e relazionale con i pubblici. Le iniziative digitali di tipo educativo promosse dai musei, in gran numero destinate al target 'bambini e famiglie', hanno fatto emergere sorprendenti capacità resilienti degli organismi museali, che si sono mostrati proattivi riuscendo a ritessere la trama della relazione con i pubblici, e hanno consentito di tracciare nuovi futuri scenari per il settore della didattica e in particolare per la didattica museale e dell'educazione al patrimonio culturale.

\section{LA DIDATTICA SENZA CONFINI: IL SETTORE MUSEALE}

Gli studi sull'uomo hanno rivelato come l'azione antropica abbia da sempre mostrato, con straordinaria continuità, soluzioni capaci di trasformare la sfuggente 
e caotica realtà in finiti modelli di discretizzazione fondati su processi cognitivi di semiosi, cioè di interpretazione e attribuzione di significati per mezzo del linguaggio. L'uomo ha potuto dunque aggredire simbolicamente la realtà per mezzo del linguaggio, rendendosi tuttavia 'incautamente' responsabile, attraverso processi cognitivi fondati su meccanismi dicotomici e oppositivi, del fiorire di arbitrarie gerarchie che hanno riguardato ogni aspetto dell'umano scibile, incluso l'ambito educativo. Come per la dimensione figurativa in cui aspri dibattimenti hanno visto contrapporre storicamente l'arte minore all'arte maggiore, le arti applicate all'arte pura, l'arte popolare all'arte egemone, allo stesso modo in ambito educativo, la concezione secondo cui il lavoro intellettuale si pone in posizione contraria a quella del lavoro manuale, sembra avere contraddistinto la storia pedagogica tout court e in particolare la relazione tra pedagogia e didattica. Basti ricordare, ad esempio, la riflessione proposta da John Dewey in ordine al valore del lavoro manuale e della pratica come processo conoscitivo e trasformativo che si realizza attraverso l'esperienza della realtà (cfr. Dewey, 2012). Al riguardo, osservano Fiorucci e Lopez, che Dewey sottolinea come:

le filosofie hanno stabilito, nel corso del loro svolgersi storico, rigide separazioni tra alcuni elementi artificiosamente posti in contraddizione tra loro: il lavoro intellettuale e il lavoro manuale, gli aspetti teorici e gli aspetti pratici della conoscenza, la conoscenza razionale e l'esperienza sensibile; in ultima istanza, la mente e il corpo. Dewey è per una concezione di educazione che superi questi dualismi e che consideri l'individuo nella sua totalità e nella sua continuità con l'ambiente che lo circonda. In questo senso egli sostiene che l'educazione può essere 'direzione' dell'educando, non nel senso di imposizione di un controllo fisico esterno su di esso, ma nel senso di assecondamento delle tendenze già presenti dentro di lui, che hanno bisogno di essere socializzate (2017, p. 10).

Il superamento del dualismo teoria versus pratica ha prodotto quel processo di 'riconoscimento' della didattica, o come si preferisce chiamare in ambito anglosassone delle 'Theories of teaching and learning', quale disciplina capace di rivendicare uno statuto epistemologico autonomo e coerente. Tale processo è stato notoriamente accompagnato nel corso del tempo da profonde trasformazioni economiche, produttive e tecnologiche che hanno mutato la nostra società, deflagrando i confini entro i quali la didattica era storicamente reclusa e ampliando definitivamente, e senza che si possa più tornare indietro, gli ambiti in cui tale disciplina si trova oggi legittimamente ad operare. Non sorprende, pertanto, che le dimensioni del lifelong e del lifewide learning impongano di decostruire quegli schemi disciplinari che per con- suetudine hanno finito per operare una spartizione simbolica degli spazi della conoscenza a favore della valorizzazione dei contesti dell'apprendimento (cfr. Commissione Europea, 2001).

In Europa, i documenti comunitari [...] fanno diffusamente riferimento a una tripartizione tra contesto formale, non formale e informale. L'apprendimento formale è quello che si sviluppa in percorsi organizzati da istituzioni quali scuole, università ed enti di formazione accreditati e che conduce all'acquisizione di certificazioni (diplomi, attestati, qualifiche e titoli). L'apprendimento non formale si verifica al di fuori delle strutture formative canoniche, ad esempio nel quadro delle attività promosse da organizzazioni e gruppi della società civile [...] L'apprendimento informale, infine, si verifica in maniera incidentale e non intenzionale nel corso della vita quotidiana, così che, sovente, non è riconosciuto come tale neppure dal soggetto stesso (Bonaiuti, 2016, p. 114).

La didattica, oramai non più "ruota di scorta della pedagogia" - come definita da Frabboni (1999) - o sorella minore, si conferma dunque, al variare dei contesti di apprendimento, una disciplina senza confini capace di recare con sé infinte opportunità teoretiche e illimitate proposte pratiche. L'attenzione all'apprendimento (learning) e alle teorie e pratiche dell'insegnamento (teaching), entrambi processi attivi che impegnano diversamente i soggetti coinvolti in una relazione educativa che è reciprocamente trasformativa, si estende così ad ambiti e settori diversi da quello scolastico, rinnovando e arricchendo sempre più la disciplina chiamata ad assumere tratti di specificità sulla base dei contesti in cui viene a trovarsi.

Con riferimento particolare al settore museale, appare utile ricordare l'attuale definizione di museo proposta e adottata dall'International Council of Museums (ICOM) nel corso della ventiduesima Assemblea Generale del 24 agosto 2007. ${ }^{3}$ Secondo la formulazione di ICOM, recepita dall'Italia con D. M. del MIBAC il $23 / 12 / 2014$, «A museum is a non-profit, permanent institution in the service of society and its development, open to the public, which acquires, conserves, researches, communicates and exhibits the tangible and intangible heritage of humanity and its environment for the purposes of education, study and enjoyment» (ICOM, 2007, p. 3). Tale enunciazione mostra con tutta evidenza

\footnotetext{
${ }^{3}$ Con riferimento alla definizione di museo proposta da ICOM, si segnala che la stessa è stata oggetto di ampia discussione in occasione della Conferenza Generale di ICOM di Kyoto 2019 ed è attualmente oggetto di revisione da parte del Committee on Museum Definition, Prospects and Potentials. Ne consegue pertanto che, ad oggi, la definizione in vigore è ancora quella adottata dall'Assemblea Generale il 24 agosto 2007.
} 
come l'educazione costituisca una delle finalità specifiche del museo che opera in qualità di istituzione in grado di contribuire allo sviluppo e alla crescita della società e dei suoi individui, legittimando in tal modo la riflessione didattica e pedagogica in ambito museale. Nel settore museale, la didattica svolge infatti un ruolo importante di mediazione culturale che «serve ad avvicinare il visitatore all'oggetto musealizzato» (Nardi, 2011, p. 24). Il valore della didattica museale, quale didattica di settore che si avvale delle indicazioni metodologiche della didattica generale, trova conferma nella posizione di Antonella Nuzzaci secondo la quale «La didattica museale [...] non si configura come attività a latere, ma come disciplina autonoma e concreta possibilità di condurre il fruitore ad acquisire quegli strumenti che gli consentano di decodificare specifiche realtà "oggettuali" e ad usare il museo quale mezzo per elaborare codici e sistemi di interpretazione della realtà» $(2012$, p. 126). Si osser$\mathrm{vi}$, al riguardo, che la complessità che questa disciplina reca con sé è riconducibile alla pluralità di settori in cui opera. Ne consegue pertanto che la didattica museale tende a configurarsi non solamente come didattica di settore ma come didattica di settori, dal momento che essa è diversamente declinata al variare della specificità di ambito dei singoli musei: archeologico, demoetnoantropologico, di arte contemporanea, di scienze naturali etc. Entro questa cornice di riferimento, a partire dalle teorizzazioni metodologiche offerte della didattica generale - che costituiscono i principi ispiratori e generativi delle pratiche educative nei musei - la didattica museale sembra potere ambire a un'autonomia teoretica per la definizione di proposte educative specifiche e caratterizzate. Si tenga in considerazione, inoltre, che la didattica museale non corrisponde sempre e in ogni caso a ciò che è comunemente inteso come "didattica" nei musei. Ciò ha generato e continua a generare spesso fraintendimenti tra ciò che è propriamente inteso come pratica didattica e le altre attività che presentano ricadute anche sul versante educativo. A nostro parere, due sono le questioni da affrontare per chiarire meglio il problema: la prima relativa al metodo e l'altra relativa al contesto. Con riferimento al metodo, il fraintendimento è causato dal fatto che le attività proposte dai musei, anche quando sono opportunamente progettate, non sempre rispondono alle funzioni fondamentali della comunicazione didattica che deve: «favorire la disposizione affettiva verso l'apprendimento; trasmettere le conoscenze necessarie; consentire il consolidamento; assicurare la verifica; predisporre una compensazione individualizzata» (Nardi, 2011, p. 69-70). Infatti, la stessa autrice sottolinea che:

Perché si possa parlare di proposta didattica in senso stretto, occorre che siano progettate e messe in atto anche le funzioni successive: non basta trasmettere conoscenze, ma occorre proporre attività che consentano di fissare tali conoscenze; non basta esporre concetti, ma occorre anche verificare la ricaduta che tale messaggio formativo ha avuto sui destinatari; non basta una proposta indifferenziata, ma occorre mettere a punto strategie che consentano di aggiustare il tiro e di compensare difficoltà individuali (Nardi, 2011, p. 71).

Con riferimento alla questione del contesto, il fraintendimento è legato al problema del riconoscimento delle pratiche museali come pratiche didattiche tout court: infatti la didattica nei musei include oggi pratiche di apprendimento finalizzate alla conservazione, fruizione e valorizzazione dell'oggetto musealizzato e non necessariamente alla decodifica in quanto bene archeologico, storico-artistico, scientifico-tecnologico etc..

Stante la specificità dell'oggetto musealizzato e del contenitore, sul piano didattico non poche risultano le difficoltà nel collocare il contesto di apprendimento nella convenzionale tripartizione formale, non formale e informale. Se, dunque, non genera dubbi il fatto che l'attività promossa dai musei possa essere ascritta entro l'ambito dell'apprendimento non formale e dell'informale - nel primo caso con riferimento alle visite guidate e ai cicli di conferenze e nel secondo caso in relazione all'azione dell'agency richiamata da Gell (1998) - la situazione si complica sul piano dell'apprendimento formale. Sempre più spesso, difatti, i musei sono promotori in autonomia o in partnership di percorsi formativi organizzati e strutturati la cui frequenza prevede il rilascio di attestati o certificati di competenza utilmente riconosciuti tanto dai soggetti privati quanto da quelli pubblici. È questo il caso, tra altri, di percorsi di formazione specialistica che prevedono l'acquisizione di crediti formativi universitari. ${ }^{4}$

Dal momento che l'apprendimento che avviene nel contesto museale non solleva i formatori dalla responsabilità che discende dal rigore imposto della pratica educativa (qualunque essa sia, in qualunque luogo essa prenda forma e qualunque siano i suoi destinatari), ne conse-

\footnotetext{
${ }^{4}$ Esempi di queste proposte educative interstiziali che sembrano collocarsi all'interno di ciò che è comunemente inteso come contesto formale di apprendimento sono: i corsi di alta formazione promossi dal MAXXI di Roma; i master universitari di II livello promossi negli aa. 2010/2011 e 2011/2012 dall'Università di Palermo in partenariato con il Museo Internazionale delle Marionette "A. Pasqualino"; i corsi di formazione dei Musei Civici di Reggio Emilia (ente accreditato dal Dipartimento Istruzione della Provincia di Reggio Emilia nell'ambito della formazione del personale insegnante della scuola); i corsi di formazione erogati dal MUSE di Trento (soggetto accreditato dal MIUR a svolgere iniziative di formazione professionale per gli insegnanti); le attività di formazione del Museo Nazionale della Scienza e della Tecnologia "Leonardo da Vinci" di Milano (soggetto iscritto all'albo regionale dei soggetti accreditati per i servizi di istruzione e formazione professionale).
} 
gue che una didattica che intenda guardare oltre le mura dell'aula scolastica deve essere capace di implementare strategicamente quelle competenze ermeneutiche necessarie a valorizzare nell'esperienza umana dell'apprendimento l'equilibrio delle dimensioni di processo e di prodotto.

\section{LE TECNOLOGIE PER LA FRUIZIONE DEL PATRIMONIO CULTURALE E L'EDUCAZIONE ONSITE E ONLINE}

Il patrimonio culturale si propone come una parte funzionalmente integrante di quell'articolato apparato di segni che caratterizza il paesaggio, inteso come conversione linguistica, logica e ideologica dello spazio (cfr. Buttitta, 1999).

Presente di un passato più o meno lontano, memoria, materiale e immateriale, di altri tempi, il patrimonio non è altro che quella parte di un tutto che ogni tempo e ogni società stabiliscono di volere proteggere e conservare per il valore simbolico che esso ha, nel suo insieme e nelle sue singole parti, come testimonianza immateriale o materiale di civiltà, allo scopo di trasmetterla alle generazioni future (Jallà, 2017, p. 10).

Questo modo di intendere le cose, secondo cui gli oggetti patrimoniali rappresentano i possenti bastioni di uno spazio antropico caratterizzato dal dominio culturale, estende la responsabilità attribuita ai musei e agli istituti culturali ed impone che essi siano coinvolti non solamente nei processi di collezione, conservazione e valorizzazione del patrimonio ma anche in azioni di empowerment per la creazione di relazioni e opportunità da offrire ai visitatori in termini di crescita personale (cfr. Morse, 2020; Del Gobbo et al., 2018; Nardi, 2011; Nuzzaci, 2012). I musei, i parchi archeologici e i complessi monumentali, funzionando come istituzioni di riferimento per le comunità, rappresentano nella loro eterogeneità tipologica quella che è possibile definire l'armatura culturale del nostro territorio-spazio-paesaggio. L'istituzione museale, senza tralasciare l'originario compito di tutela e salvaguardia, è chiamata a partecipare alle complesse politiche di sviluppo sostenibile enfatizzando la relazione tra gli oggetti museali e i loro contesti di provenienza (i paesaggi culturali di cui sono espressione), sviluppando un confronto con le visioni patrimoniali delle comunità, integrando la prospettiva museum oriented con quella context oriented (cfr. Jallà, 2017). Con riferimento alle pratiche didattiche che hanno come oggetto il patrimonio culturale, il superamento di posizioni object-centred produce «un passaggio da attività esclusivamente destinate a trasferire "specifiche" conoscenze relative ad un oggetto a audience "specifiche" come studenti, famiglie o adulti, ad attività alternative attente ai bisogni di particolari gruppi o soggetti» (Del Gobbo, 2018, p. 26). In tal senso, le istituzioni patrimoniali svolgono una concreta missione sociale all'interno dei territori in cui si trovano ad operare, ora fornendo soluzioni ai bisogni sociali, ora promuovendo l'educazione permanente attraverso la creazione di nuove comunità interpretative che assumono il museo e i luoghi del patrimonio come spazi della condivisione per sperimentare pratiche di didattica per il patrimonio socialmente rilevanti. L'azione educativa del patrimonio, scrive Chiara Panciroli,

non consiste solo nella comunicazione dei contenuti culturali e simbolici, materiali e non materiali, formali o informali, in presenza e a distanza o ancora nell'insieme di eventi-iniziative atte a consolidare il rapporto fra pubblico-oggetti-strutture museali, ma soprattutto come riscoperta dell'identità dell'uomo e della comunità di appartenenza, di cui il patrimonio ne è espressione (2014, pp. 21-22).

Dal momento che il patrimonio culturale può essere considerato anche uno strumento funzionale per la comunicazione di contenuti simbolici, esso può essere utilmente impiegato per promuovere azioni di educazione socialmente rilevanti per la società contemporanea, fortemente connotata dalla dimensione della variabilità (culturale, sociale, economica, linguistica, etc.). D’altra parte, scrive Antonella Poce, «il patrimonio artistico e culturale si presta perfettamente come luogo di sollecitazioni ed esercizio di quelle abilità utili all'adattamento in un contesto in continua mutazione, quale quello contemporaneo» (2017, p. 11). In particolare, tra i luoghi di fruizione del patrimonio culturale, il museo sembra funzionare efficacemente come facilitatore dello sviluppo di pensiero critico perché esso è a un tempo spazio di significazione, in quanto dispositivo e messaggio di un processo di comunicazione e a un altro, luogo di negoziazione in cui le soggettività sono inevitabilmente opposte (ad esempio, il curatore vs il fruitore).

Alla fine del secolo precedente il modello che caratterizzava la fruizione del patrimonio all'interno del museo era di tipo trasmissivo, in cui l'utente assumeva un ruolo pressoché passivo. Nel corso dei decenni più recenti, si è assistito a una sempre maggiore attenzione al processo esperienziale di cui è protagonista il visitatore del museo. Tale interesse, ancorché risponda inizialmente in modo esclusivo a logiche volte a sviluppare nuove strategie di audience development, è oggi l'esito congiunto tanto della riflessione promossa dagli studi sul 
cultural heritage quanto di quella sollecitata delle scienze dell'educazione che concordano nel ritenere il museo uno spazio democratico e funzionale in cui è possibile costruire efficaci pratiche di insegnamento e apprendimento. Riguardo la significatività dell'apprendimento in ambito museale, osserva Hein, "the most important issues involved in understanding learning are derived from analyzing the actions of the learner rather than in probing the nature of the subject to be learned» (1995, pp. 189-190).

Se, secondo la vecchia concezione elaborata nell'ambito delle teorie museologiche di fine Ottocento, l'oggetto museale possiede per sua natura le caratteristiche di 'cosa per essere mostrata', pensato e progettato per essere esposto (dipinti, statue, arazzi, manufatti etc.), per intrattenere ma anche per istruire ed emozionare, l'attuale riflessione museale, mettendo in discussione il rapporto tra materiale e immateriale, celebra il valore di medium dell'oggetto che si fa semioforo (cfr. Pomian,1978). Ne consegue che l'oggetto non costituisce più la realtà stricto sensu ma assume il valore che la comunità gli attribuisce, riducendo e talvolta negando l'idea che l'oggetto museale coincida necessariamente con l'oggetto patrimoniale. Nell'ambito di tale scenario, un ruolo decisivo è stato svolto dall'introduzione in ambito museale e patrimoniale della tecnologia. L'invasione della tecnologia, miniaturizzata, integrata, biocompatibile interconnessa con il corpo è una realtà che esperiamo nella pratica quotidiana e che ci obbliga a riavviare criticamente la 'macchina cognitiva' per ripensare i processi di accesso alla realtà che non avverranno più esclusivamente in intellectu ma in peritia, cioè nella tecnica acquisita con l'uso della tecnologia. Lo scostamento simbolico e il graduale allontanamento tra il piano museale e patrimoniale dell'oggetto, tra cosa e processo, tra visibile e invisibile, tra semplice osservazione contemplativa e performance esperienziale sono stati possibili grazie all'introduzione sempre più massiva in campo museale di macchine e strumenti tecnologici che, svelando i limiti organici dell'uomo, intervengono su di esso modificandone il corpo. L'uomo viene 'dopato tecnologicamente' attraverso processi di riprogettazione corporea finalizzati a dotarlo di abilità neuro-cognitive e capacità sensoriali sovrumane o meglio, post-umane (cfr. Stelarc, 1994). È il caso, ad esempio, del ricorso alla tecnologia, sempre più frequente negli allestimenti, a realtà aumentata, superfici interattive, diffusioni olfattive, specchi virtuali e schermi olografici, touch e multi-touch, guide avatar, totem e in genere a tutti quegli strumenti che da un lato presuppongono un pacifico assoggettamento del corpo all'esperienza museale e dall'altro enfatizzano l'assenza della presenza oggettuale restituendo talvolta effi- cace ricostruzione della natura ipermediale del messaggio museale.

Senza entrare nel merito delle implicazioni riconducibili all'Embodied Cognitive Science (cfr. Steier, Pierroux \& Krange, 2015), esempi ineludibili di come l'esperien$\mathrm{za}$ in ambito museale possa svilupparsi in una dialettica tra corpo e tecnologia sono rappresentati dalle metodologie immersive (realtà virtuale; realtà aumentata; realtà mista). Con riferimento al potenziale che la tecnologia mette a disposizione per la partecipazione attiva all'esperienza di apprendimento al museo, in un recente articolo Silvia Coppola e Silvia Zanazzi sottolineano che:

i musei costituiscono un luogo di aggregazione e di interazione tra persone molto diverse tra loro sia per età che per contesto socio-culturale: di conseguenza, è difficile progettare attività adeguate a tutti i target e che abbiano la stessa risonanza in ogni visitatore che le prova. Per garantire un'esperienza soddisfacente, quindi, è opportuno pensare a soluzioni specifiche per determinate categorie. Uno dei maggiori vantaggi dell'uso delle tecnologie in ambito museale, riconosciuto nella letteratura, è proprio la "personalizzazione": il visitatore può scegliere tra una varietà di opzioni in base alle sue preferenze, desideri e possibilità (2020, p. 44).

Le pratiche educative nei musei si servono oggi in maniera sempre più massiva delle tecnologie digitali le cui potenzialità consentono di sviluppare nuove narrazioni a partire, ad esempio, dalla biografia dell'oggetto musealizzato e dal contesto in cui è stato prodotto. La tecnologia dunque si conferma da una parte come risorsa capace di attivare innovative risonanze tra il museo e il destinatario dell'azione educativa e dall'altra come utile strumento di benchmarking dei desideri dei pubblici. È utile ricordare, con riferimento alle pratiche di educazione museale destinate ai bambini e ai ragazzi, che l'utilizzo della tecnologia appare spesso una condizione necessaria per il coinvolgimento di quel target che comunemente indichiamo come 'nativi digitali' e che mostra, anche in ambito museale, una sempre maggiore preferenza al consumo di prodotti tecnologici e digitali sia onsite che online. Se è vero che l'enfasi della presenza fisica in ambito museale è sempre stata molto forte - basti ricordare il valore attribuito all'esperienza artistica nell'ambito del percorso formativo e iniziatico che i giovani di buona famiglia intraprendevano con il grand tour già dal XVII secolo - l'impiego del digitale e delle tecnologie, per la fruizione o per l'apprendimento al tempo del Covid-19, ha fatto emergere una nuova relazione tra il museo (i suoi contenuti e i suoi oggetti) e i suoi pubblici. L'esperienza prodotta dall'isolamento imposto ai cittadini per il contenimento della diffusione dell'epidemia da Covid-19, sembra indurre a un legitti- 
mo ripensamento dell'Interactive Experience Model presentato da Falk e Dierking (cfr. Falk \& Dierking, 1992). Difatti, al personal context, social context e physical context, sembra potersi aggiungere opportunamente un quarto elemento che è capace di generare contenuti mutevoli e imprevedibili nella relazione museale e cioè il 'digital and technological context'. L'interazione di questa tetrade sembra potere rappresentare oggi l'inesauribile linfa della narratologia museale (cfr. Panciroli, 2019).

\section{IL MUSEO AL TEMPO DEL COVID-19. LOCKDOWN E ASSEMBRAMENTO DIGITALE}

L'emergenza epidemiologica generata dalla diffusione pandemica dell'infezione Covid-19 ha coinvolto settori e ambiti diversissimi tra loro e ha indubitabilmente prodotto una nuova modalità di percepire il rischio connesso alla perdita della salute da parte di tutti i cittadini. Se la comunicazione scientifica e la divulgazione di qualità impegnate a contrastare l'infodemia hanno svolto un ruolo cruciale in quel processo trasformativo che ha indotto il ripensamento delle nostre società, i provvedimenti assunti dai governi per rispondere all'emergenza sanitaria hanno obbligato a riorganizzare attività e processi, attraverso lo sviluppo di soluzioni volte al superamento della crisi pandemica. In un così complesso e inaspettato scenario i media e il digitale hanno riaffermato il loro valore, quali validi strumenti coadiuvanti l'agire umano tanto in presenza quanto in assenza.

In Italia, l'emanazione del Decreto del Presidente del Consiglio dei Ministri dell'8 marzo 2020, relativo all'adozione di misure urgenti per il contrasto e il contenimento del contagio da Covid-19, ha disposto la sospensione e la chiusura immediata di numerose attività ritenute non essenziali. In conseguenza di tale provvedimento, il già fragile settore dell'educazione e della cultura è stato protagonista di un improvviso arresto. Con specifico riferimento al settore museale, l'art. 2, comma 1 del citato D.P.C.M. indicava che: «è sospesa l'apertura dei musei e degli altri istituti e luoghi della cultura di cui all'articolo 101 del codice dei beni culturali e del paesaggio, di cui al decreto legislativo 22 gennaio 2004, n. 42» (D.P.C.M. 8 marzo 2020). I musei, le cui alterne fortune sono riconducibili il più delle volte alla capacità che essi hanno nel costruire azioni sinergiche all'interno del territorio in cui si trovano ad operare, già fortemente provati dalla stretta finanziaria che da anni procede nella direzione di una vigorosa spending review, hanno dovuto recidere, seppur temporaneamente, il legame forte con i propri pubblici, interrompendo l'azione compartecipativa che costituisce le relazioni di senso con gli oggetti museali. Al riguardo, una recente indagine ISTAT sui musei statali italiani ha fatto emergere che:

Basandosi sulla serie storica dei dati forniti dal Ministero è possibile stimare che per il 2020, in assenza di Covid19, si sarebbe potuto realizzare un incremento del numero di visitatori dei musei statali dell' $8,1 \%$ rispetto al 2019 e un aumento degli introiti lordi del 12,8\%. Per il 2020, infatti, si può stimare che il pubblico dei musei, dei monumenti e delle aree archeologiche a gestione statale sarebbe stato complessivamente di 59,2 milioni di visitatori, e che avrebbe permesso di realizzare entrate per oltre 273 milioni di euro. A seguito della chiusura al pubblico necessaria per il contenimento del contagio da Covid-19, si stima invece che per i soli mesi di marzo, aprile e maggio di quest'anno il numero di mancati ingressi alle strutture statali sia stato pari a circa 19 milioni, con una perdita, in termini di mancati incassi, di circa 78 milioni di euro (ISTAT, 2020, p. 4).

Se il lockdown ha impedito l'accesso fisico al contenitore museale, vietando la fruizione del patrimonio culturale ai fini dell'edu-info-entertainment, esso ha generato anche nel settore della cultura una significativa accelerazione a quel processo di innovazione digitale che tarda ancora a realizzarsi pienamente in Italia. ${ }^{5}$ Infatti, dopo alcuni giorni di vacuum, in cui le istituzioni culturali ancora disorientate dalla sospensione imposta dal governo hanno dovuto rifondare le proprie attività, si è assistito in modo graduale ma costante a una sempre maggiore effervescenza digitale e a un diffuso impiego dei social e del web. All'impossibilità di incontrarsi in presenza, $\mathrm{i}$ musei e i luoghi della cultura hanno risposto con la conquista massiva di un nuovo spazio, quello del web, in cui era consentito riunirsi, condividere e riallacciare il legame con il territorio e con il pubblico. L'assembramento digitale, l'unico possibile al tempo del Covid-19, ha prodotto innumerevoli occasioni di incontro per dialogare con le comunità e una vera e propria esplosione del confronto online tra studiosi ed esperti dei settori scientifici più disparati che hanno preso parte a webinar, conference call, mooc etc. A tale riguardo, Agostino, Arnaboldi e Lampis sottolineano che: «Social media platforms, especially Facebook, Twitter and Instagram, have become the museums' preferred means to spread culture during the COVID-19 lockdown. Museums physically shut to the public were instead open because of their digital tools.

\footnotetext{
${ }^{5}$ È opportuno segnalare che, coerentemente con quanto previsto dalla Riforma del MIBAC 2014, con il Decreto del Direttore della Direzione Generale dei Musei n. 892 del 19/07/2019 è stato approvato in Italia il primo "Piano Triennale per la digitalizzazione e l'Innovazione dei Musei" con il fine, tra gli altri, di «Attivare nuove forme di accesso e fruizione dei dati relativi al patrimonio, mediate o abilitate da soluzioni tecnologiche» (MIBACT, 2019, p. 9).
} 
And they were not only open, they also saw a significant rise in their online activity» $(2020$, p. 5). Si osservi, inoltre, che prima della diffusione dell'epidemia da Covid-19 il livello di innovazione digitale dei musei italiani presentava ampi margini di miglioramento e il digitale era nella più parte dei casi impiegato come uno strumento complementare e di supporto alla visita in loco. Il Covid-19, imponendo una trasformazione delle modalità di consumo del prodotto culturale, cambia la percezione del digitale che diviene l'unico mezzo per mantenere viva la stessa istituzione museale. L'isolamento forzato imposto dal lockdown e lo schizofrenico ricorso al web da parte delle istituzioni museali, al fine di riallacciare la relazione con il pubblico, ha prodotto in Italia esiti estremamente variabili e ciò anche in conseguenza del differente grado di competenza digitale delle risorse umane impiegate all'interno di ciascun museo. Dal momento che l'utilizzo dei media digitali non corrisponde sempre e in ogni caso al possesso delle competenze digitali, le uniche necessarie a disvelare secondo un approccio eticamente critico i vizi e le virtù del digitale (cfr. Rivoltella, 2015), il dibattito scientifico ha fatto emergere alcune delle criticità più evidenti:

A first critical issue is linked to mediation and authority over content. [...] A second important issue relates to the organisational change necessary for managing social media, which requires new skills and processes (Giannini \& Bowen, 2019; Silvaggi \& Pesce, 2018). The third and final issue that emerged clearly during the COVID-19 pandemic is for social media to be inserted within a wider user experience, where the boundaries between physical and digital worlds become seamless, according to the new concept of 'phygital' (physical + digital) experience (Ballina, Valdes, \& Del Valle, 2019) (Agostino et al., 2020, p. 3).

La prima questione appare direttamente riconducibile all'abilità che un consumatore dei media digitali avveduto sviluppa nel dubitare dell'affidabilità e dell'autorevolezza della fonte (cfr. Rivoltella, 2015); la seconda criticità, che riflette in ambito museale una visione skill-based delle competenze digitali quali acquisizioni medium related cioè operazionali e formali del singolo soggetto (cfr. Greco \& Caria, 2020), attiene alle professionalità che lavorano all'interno dell'istituzione museale e infine, la terza criticità riguarda il ricorso a metodologie immersive (realtà mista) capaci di integrare risorse fisiche e digitali per un processo di co-creazione dell'esperienza museale (cfr. Nofal et al., 2017).

A conferma del fatto che l'aumento dell'utilizzazione del digitale da parte dei musei non rappresenta un trend esclusivo italiano, appare utile riportare alcuni dati significativi emersi da un'indagine internazionale condotta dal
Network of European Museum Organisations (NEMO) tra il mese di marzo e quello di aprile 2020. La 'Survey on the impact of the COVID-19 situation on museums in Euro$p e^{\prime}$ (cfr. NEMO, 2020), che ha coinvolto circa 1000 istituzioni museali presenti in 48 stati (27 U.E. e 21 non U.E.), ha rivelato che Almost $70 \%$ of the museums increased their online presence since they were closed due to social distancing measures. After 3 weeks of closure to the public, already $80 \%$ of the museums had increased their online activity, reacting to the general increased visibility of digital cultural heritage on the internet» (NEMO, 2020, p. 12). In particolare, si mostra come un aspetto estremamente rilevante il fatto che i musei abbiano confermato il loro ruolo di presìdi educativi anche a distanza promuovendo quiz, giochi, attività di vario genere e fornendo materiali didattici. A tale proposito, mostra l'indagine NEMO, «Museums reported that next to social media, online services such as educational material are most popular with online visitors, followed by videos and films, viewing the collection, and behind the scenes materials» (NEMO, 2020, p. 16).

Il lockdown imposto dalla pandemia 2020 restituisce dunque, tanto nel contesto italiano quanto in quello europeo ed extraeuropeo, l'immagine di un museo al tempo del Covid-19 proattivo e resiliente che da una parte desidera riconnettersi con il territorio e con i pubblici attraverso il web e dall'altra progetta strategie per sfruttare le potenzialità dei media digitali, al fine di indagare la storia e la biografia degli oggetti attraverso pratiche di fruizione ed educazione attiva, fondate sul principio della personalizzazione e dell'interazione.

\section{LA RESILIENZA DELL'ORGANISMO MUSEALE. ALCUNE INIZIATIVE DIGITALI}

Come indicato in precedenza, l'indiscusso valore del patrimonio culturale risiede non solo nella capacità di rappresentare la storia e la cultura di un territorio ma si esplica anche e soprattutto nella capacità di offrirsi quale pretesto per dialogare con le comunità di riferimento, per sviluppare azioni di empowerment e per promuovere soluzioni creative ai vecchi e nuovi problemi. Ne consegue, come osservano Del Gobbo, Torlone \& Galeotti, che «Il Post-museo si configura [...] come esperienza e processo educativo (più che come modello di infrastruttura), condivisi con le comunità territoriali in cui il museo è insediato. Esso è chiamato a offrire contesti stimolanti, che incoraggino lo sviluppo di nuovi saperi (sia del pubblico che dei curatori museali)» (2018, p. 75) e che tengano conto della zona di sviluppo prossimale tanto del destinatario dell'azione didattica quanto dell'insegnan- 
te, in relazione della capacità che egli ha di sviluppare le proprie abilità e di ampliare le competenze.

Dal punto di vista didattico, appare evidente che il passaggio da uno spazio fisico a uno spazio digitale in cui ha luogo il processo di apprendimento non può avvenire senza un necessario ripensamento del cosa e del come apprendere. Infatti, l'esperienza scolastica della $\mathrm{DaD}$, che il lockdown ha imposto non più come scelta ma come condizione inevitabile, ha mostrato come fosse necessario riconsiderare le strategie d'insegnamento, l'utilizzo della tecnologia a supporto della didattica e i contenuti da selezionare per un apprendimento efficace che non sovraccarichi lo sforzo cognitivo dell'alunno. Come per la scuola, anche per i musei si è fatta strada in misura sempre maggiore la consapevolezza dell'impossibilità di replicare online modelli didattici impiegati tradizionalmente onsite. Alle limitazioni imposte dalla diffusione della pandemia da Covid-19 le istituzioni museali come detto sopra - hanno fatto ampio ricorso ai mediatori digitali e non sorprende che il Network of European Museum Organisations abbia incluso tra le proprie raccomandazioni l'invito a considerare che «digital cultural heritage and digital engagement have demonstrated its value in the past weeks by bringing people together, encouraging creativity, sharing experiences, and offering a virtual space to build ideas collectively» (NEMO, 2020, p. 3). Durante l'isolamento imposto dallo scenario pandemico, i musei e i luoghi della cultura hanno promosso molteplici iniziative digitali fondate su modelli didattici attivi come la condivisione di ipertesti, clip audio e video, narrazioni in immagini, percorsi virtuali, riproduzioni in high definition, tour 3D etc.

All the different types of material on offer (interviews, discovering artwork, quizzes, virtual visits) set in motion a range of approaches for connecting with the public. The first type is an educational approach, where material is passed by the museum to the user. [...] The second approach involves asynchronous interaction, and is when the user is handed information or material, but is not required to join in or reply on social media. Treasure hunts and ArtYouReady flashmobs fall into this group. [...] Finally, the third approach consists of synchronous interaction, where museum and visitor interact in real time. Here the relationship is two-way but, differently from the previous case, it is simultaneous. This group includes educational initiatives with real-time interaction between museum and students/children and meetings with Museum Friends (Agostino et al., 2020, p. 6).

Il ricorso alle potenzialità offerte dalla rete internet ha consentito ad alcuni importanti soggetti impegnati nel settore del patrimonio culturale di animare il web attraverso la realizzazione di pagine internet e piatta- forme virtuali, per la condivisione delle attività culturali proposte dai musei durante il lockdown. Alcuni esempi particolarmente significativi sono: "Covid-19 - How to explore art and culture and heritage of Europe from home" della divisione Culture and cultural heritage del Consiglio d'Europa (cfr. Consiglio d'Europa, 2020); "La cultura non si ferma" del Ministero per i beni e le attività culturali e per il turismo (cfr. MIBACT, 2020); le bacheche virtuali promosse sulla piattaforma Padlet da ICOM Italia ('\#laculturanonsiferma'; 'Risorse e informazioni'; 'Bambini e famiglie') (cfr. ICOM, 2020).

Con specifico riferimento al Padlet ICOM 'Bambini e famiglie', è utile segnalare che esso rappresenta uno dei segnali più evidenti del fatto che durante tutto il periodo di isolamento sia stata riservata una straordinaria attenzione al target "infanzia". Durante il lockdown, se nell'ambito dell'apprendimento formale la didattica a distanza ha reso indistinguibile lo spazio della scuola e quello della famiglia, non diversamente è accaduto per il consumo culturale ai fini del diletto o dell'educazione. Nel confuso tempo e spazio che ha caratterizzato le settimane di isolamento adulti e bambini hanno dovuto riallineare i ritmi del lavoro, dell'apprendimento e del gioco dovendo sovente affrontare rocambolesche e ardimentose sovrapposizioni. Al fine di documentare l'impegno delle istituzioni museali nel portare avanti iniziative digitali ed educative rivolte al target "bambini e famiglie" durante il periodo d'isolamento, si riportano di seguito alcune descrizioni sintetiche ed esemplificative delle numerosissime attività proposte dai musei e delle varie tipologie reperibili online:

1. \#popupcontroilcoronavirus, Museo della Scuola e del Libro per l'Infanzia di Torino. Nell'ambito di un progetto di collaborazione con pop up designer e con il sito internet www.pop-up.org, il Museo della Scuola di Torino ha promosso azioni di informazione e sensibilizzazione sul tema della lotta al Coronavirus attraverso tutorial per la realizzazione di tavole, card animate e libretti pop up. Il ricorso a immagini e template scaricabili dalla pagina Facebook ha consentito l'attivazione di processi di personalizzazione nella realizzazione del prodotto pop up e la successiva restituzione mediante la condivisione delle fotografie da parte dei destinatari dell'attività (cfr. Città di Torino, 2020).

2. \#trovalastatua, Gipsoteca di Arte Antica dell'Università di Pisa. La Gipsoteca di Pisa ha organizzato sulla propria pagina Facebook un'attività volta alla scoperta delle opere costituenti la collezione. Attraverso la pubblicazione periodica delle immagini raffiguranti le sagome delle statue, è stato proposto 
ai ragazzi uno sforzo immaginativo per pensarsi all'interno della Gipsoteca, rimasta al buio a causa del Covid-19, al fine di riconoscere le opere d'arte. Il tour virtuale del museo, promuovendo il patrimonio culturale, ha fornito il necessario supporto ai partecipanti. L'attività trovava conclusione con un momento d'interazione: a questi ultimi, infatti, è stato chiesto di inviare un messaggio privato con il nome dell'opera, ricevendo in riscontro l'immagine della statua scovata da colorare (cfr. Gipsoteca di Arte Antica dell'Università di Pisa, 2020).

3. \#ungiocodaragazzi, Museo Archeologico Nazionale di Venezia. L'hastag 'ungiocodaragazzi' lanciato dal Museo Archeologico di Venezia ha incluso una serie di iniziative tese a sollecitare la creatività dei giovani attraverso il ricorso anche ad attività manuali volte alla conoscenza della cultura classica. È il caso dei crotali, lo strumento a percussione degli antichi greci che, seguendo le indicazioni fornite dal museo con un post della pagina del social network Facebook, è possibile riprodurre utilizzando oggetti facilmente reperibili all'interno della propria abitazione come tappi di bottiglie, pennarelli, cartoncini etc. (cfr. Museo Archeologico Nazionale di Venezia, 2020).

4. \#storieaportechiuse, Museo Nazionale della Scienza e della Tecnologia Leonardo da Vinci di Milano. Il Museo della Scienza di Milano ha elaborato durante il Covid-19 il format \#storieaportechiuse che ha previsto una serie di incontri in modalità asincrona con finalità divulgative ed educative rivolte a un pubblico di bambini e ragazzi. I video, pubblicati sulla pagina del Museo e sui canali digitali (Facebook, YouTube etc.) e della durata di circa 5 minuti, presentano una specifica trattazione tematica e propongono attività facilmente realizzabili a casa. È il caso, ad esempio, del tutorial "colle rinascimentali" che, a partire dagli insegnamenti di Leonardo da Vinci sull'utilizzo delle materie prime, ha proposto la creazione di una colla fatta in casa (cfr. Museo Nazionale della Scienza e della Tecnologia Leonardo da Vinci, 2020).

5. \#talindopiccione, Museo Benozzo Gozzoli di Castelfiorentino (FI). Il 5 aprile 2020, in piena fase di lockdown, il Museo BeGo lancia sulle proprie pagine internet e Facebook e sul proprio canale YouTube una storia animata per bambini e ragazzi che ha come protagonista un piccione di nome Talindo. Al fine di conquistare la curiosità del giovane pubblico, condotto in un innovativo tour del museo, Talindo è coinvolto in un'avventura in quattro brevi episodi all'interno del percorso museale dove dialoga con $\mathrm{i}$ protagonisti delle opere, svelandone storie e segreti (cfr. Museo Benozzo Gozzoli, 2020).

6. \#iocolorolalanterna, Lanterna di Genova. Simbolo della città di Genova, la Lanterna ha lanciato alla fine del mese di marzo un contest per la scelta del colore che avrebbe illuminato la Lanterna la sera del successivo 20 aprile. Per prendere parte alla competizione online, è stato chiesto ciascun partecipante di produrre un disegno colorato della Lanterna da condividere su Facebook e Instagram unitamente a un breve post relativo alle ragioni che hanno indotto la scelta del colore. Infine, il numero dei like, quale espressione dell'apprezzamento da parte dei follower dei social, ha decretato il vincitore (cfr. Lanterna di Genova, 2020).

7. \#ilmuseoacasavostra, Civico Museo Archeologico di Camaiore $(L U)$. Per riallacciare la trama della relazione con il pubblico più giovane, il Civico Museo Archeologico di Camaiore ha organizzato durante il periodo di chiusura dirette tematiche su Facebook e YouTube volte a promuovere la conoscenza degli oggetti museali, valorizzandone la storia connessa al loro uso. È il caso, ad esempio, della diretta "a tavola con i Romani”, un tutorial per la preparazione di una focaccia il cui consumo è attestato già dal II secolo a.C.. L'attività ha previsto la riproduzione della ricetta da parte dei partecipanti all'attività e la condivisione sulla pagina Facebook del museo delle immagini relative alla preparazione e al consumo (cfr. Civico Museo Archeologico di Camaiore, 2020).

8. \#discoverymuseumchallenge, MarteS e I musei di Brescia. L'iniziativa, promossa dall'associazione "La Melagrana", ha previsto la realizzazione di una challenge rivolta ai bambini per la scoperta di dieci musei o siti culturali del territorio bresciano, attraverso giochi diversificati in base all'età. Giochi enigmistici, quiz, lavoretti da realizzare relativi ai siti culturali del territorio hanno scandito le dieci tappe della gara che si è svolta online dal 13 marzo al 3 aprile. I biglietti di ingresso ai musei e la partecipazione gratuita ad attività ludico-didattiche hanno rappresentato i premi per i vincitori della challenge (cfr. Museo d'Arte Sorlini, 2020).

9. \#dragoarturo, Maxxi di Roma. Il Museo nazionale delle arti del XXI secolo di Roma, nell'ambito delle iniziative digitali promosse per coinvolgere il pubblico più giovane, ha lanciato sulle proprie pagine social e sul proprio sito internet un tour per immagini del Museo attraverso il riutilizzo di didascalie e disegni, esiti di attività laboratoriali svolte in presenza. Il drago Arturo, mascotte del tour, ha accompagnato i bambini e i ragazzi alla scoperta online 
di alcune delle opere più importanti conservate nel museo (cfr. Museo Nazionale delle Arti del XXI secolo, 2020).

10. \#mubaleggecorraini, Museo dei Bambini di Milano. Il MUBA di Milano, che si propone come centro per lo sviluppo e la diffusione di progetti culturali dedicati all'infanzia, ha promosso durante il lockdown un ciclo di video-letture dedicate ai bambini. L'attività, condotta in collaborazione con Corraini edizioni, ha previsto la pubblicazione sul canale social di Facebook di brevi video condotti da un'educatrice in cui alla lettura si accompagnava la visione delle immagini contenute all'interno dei volumi (cfr. Museo dei Bambini di Milano, 2020).

\section{CONCLUSIONI}

La storia dell'umanità ha dimostrato che le crisi economiche, demografiche, sociali financo quelle epidemiche hanno rappresentato in passato delle straordinarie opportunità per elaborare e realizzare innovativi modelli di sviluppo con ricadute locali e globali.

Nello scenario disegnato dal Covid-19, in cui la didattica online e le iniziative digitali hanno rappresentato spesso i soli strumenti a disposizione delle agenzie educative per riallacciare la relazione bruscamente interrotta con gli allievi, la breve rassegna delle attività realizzate nel settore del cultural heritage ha dimostrato come i musei siano stati in grado di promuovere attività in ambiente virtuale con sorprendenti abilità responsive.

La relazione tra media ed educazione nell'era della pandemia di Covid-19 si connota dunque, anche in ambito museale, per le innumerevoli opportunità mediate dalle tecnologie e dall'online. L'osservazione dello sperimentalismo delle pratiche realizzate negli ultimi mesi, tanto in ambito scolastico quanto in ambito museale, ha fatto emergere come le tecnologie, il web e i social network abbiano agito da una parte come catalizzatori dei processi di valorizzazione del cultural heritage e dall'altra come corroboranti per la realizzazione di luoghi di assembramento digitale che favoriscono il dialogo, per la co-creazione di nuove soluzioni di apprendimento e per la promozione di interventi di empowerment.

Nel segno dunque di un proposito eudemonico e confidando in un investimento serio nel settore dell'educazione e del capitale umano, si è inteso proporre alcune prime considerazioni come esito di una preliminare attività di censimento delle iniziative educative promosse nel web e nei social network dai musei durante il lockdown.

Se è vero che ogni intendimento di riflessione appare sempre più complesso quando ha come oggetto un feno- meno concluso di recente, o peggio ancora nel suo svolgersi, come nel caso del Covid-19, la sistematica analisi comparativa delle soluzioni efficaci adottate dai musei in risposta alla diffusione della pandemia di Covid-19, rappresentando una delle nuove sfide del futuro della didattica e in particolare della didattica museale, consentirà l'accrescimento delle competenze necessarie nel caso di ulteriori future pandemie, comprese quelle connesse alla riprogettazione e al rimodellamento delle pratiche d'insegnamento e apprendimento all'interno dei contesti non formali come i musei e luoghi della cultura.

\section{BIBLIOGRAFIA}

Agostino, D., Arnaboldi, M., \& Lampis, A. (2020). Italian state museums during the COVID-19 crisis: from onsite closure to online openness. Museum Management and Curatorship, 1-11. https://doi.org/10.1080/0 9647775.2020 .1790029

Bonaiuti, G. (2016). Gli ambiti della didattica. In G. Bonaiuti, A. Calvani \& M. Ranieri (eds.) (2016). Fondamenti di didattica: Teoria e prassi dei dispositivi formativi, 113-144. Carocci editore.

Buttitta, A. (1999). Il principe e la memoria di un paesaggio immemoriale in G. Cusimano (ed.) (1999). $\mathrm{La}$ costruzione del paesaggio siciliano: geografi e scrittori a confronto. Università degli Studi di Palermo.

Citta di Torino (2020). POP UP contro il coronavirus. http://www.comune.torino.it/museiscuola/app/popup-contro-il-coronavirus.shtml

Civico Museo Archeologico di Camaiore (2020). A tavola con i romani!. https://youtu.be/ykwKkJiiZxw

Consiglio d'Europa (1998). Recommendation of the Committee of Ministers to member Statesconcerning heritage education. https://rm.coe.int/16804flcal

Consiglio d'Europa (2017). Recommendation of the Committee of Ministers to member States on the European Cultural Heritage Strategy for the 21st century. https://rm.coe.int/16806f6a03

Consiglio d'Europa (2020). Covid-19 - How to explore art and culture and heritage of Europe from home. https://www.coe.int/en/web/culture-and-heritage/ access-to-culture-during-covid-19

Commissione Europea (2001). Realizzare uno spazio europeo dell'apprendimento permanente. https:// archivio.pubblica.istruzione.it/dg_postsecondaria/ allegati/apprperm211101.pdf

Coppola, S., \& Zanazzi, S. (2020). L'esperienza dellarte: il ruolo delle tecnologie immersive nella didattica museale. Formazione \& Insegnamento, 18(2), 36-49. https://doi.org/10.7346/-fei-XVIII-02-20_04 
Crowley, K., Pierroux, P., \& Knutson, K. (2014). Informal learning in museums. In R. K. Sawyer (ed.) (2014). The Cambridge handbook of the learning sciences, 461-478. Cambridge University Press.

Del Gobbo, G. (2018). Un paradigma inclusivo per il patrimonio culturale. In G. Del Gobbo, F. Torlone \& G. Galeotti (eds.) (2018). Le valenze educative del patrimonio culturale. Riflessioni teorico-metodologiche tra ricerca evidence based e azione educativa nei musei, 25-58. Aracne editrice.

Del Gobbo, G., Torlone, F., \& Galeotti, G. (eds.) (2018). Le valenze educative del patrimonio culturale. Riflessioni teorico-metodologiche tra ricerca evidence based $e$ azione educativa nei musei. Aracne editrice.

Dewey, J. (2012). Democrazia e educazione. Sansoni.

D.P.C.M. 8 marzo 2020. Ulteriori disposizioni attuative del decreto-legge 23 febbraio 2020, n. 6. Gazzetta Ufficiale della Repubblica Italiana. Serie Generale, n. 59.

Falk, J. H., \& Dierking L. D. (1992). The museum experience. Whalesback Books.

Fiorucci, M., \& Lopez, G. (2017). Introduzione in M. Fiorucci, G. Lopez (eds.) (2017). John Dewey e la pedagogia democratica del '900, 7-17. RomaTre-Press.

Frabboni, F. (1999). Didattica generale: una nuova scienza dell'educazione. Bruno Mondadori.

Gell, A. (1998). Art and agency: an anthropological theory. Clarendon Press.

Gipsoteca di Arte Antica dell'Università di Pisa (2020). \#trovalastatua. https://www.facebook.com/gipsotecapisa

Greco, G., \& Caria, M. (2020). Competenze digitali per la media education: il modello blended learning di Monopoli. Media Education, 11(1), 25-35.

Hein, G. E. (1995). Evaluating teaching and learning in museums in E. Hooper-Greenhill (ed.) (1995). Museum, media, message (pp. 189-203). Routledge.

ICOM (2007). ICOM Statutes. https://icom.museum/wpcontent/uploads/2018/07/2017_ICOM_Statutes_EN.pdf

ICOM (2020). Padlet. https://it.padlet.com/IcomItalia

ISTAT (2020). I musei italiani al tempo del Covid-19. https://www.istat.it/it/files//2020/05/I-musei-statalial-tempo-del-Covid-19.pdf

Jallà, D. (2017). Paesaggi culturali e musei: il museo nella sua quarta età. Museo in forma, 59 (XXI), 9-18.

Lanterna di Genova (2020). Colora la tua lanterna. https://www.lanternadigenova.it/colora-la-tua-lanterna/

MIBACT (2019). Piano Triennale per la Digitalizzazione e l'Innovazione dei Musei. http://musei.beniculturali. it/wp-content/uploads/2019/08/Piano-Triennale-perla-Digitalizzazione-e-1\%E2\%80\%99Innovazione-deiMusei.pdf

MIBACT (2020). La cultura non si ferma. https://www. beniculturali.it/laculturanonsiferma (ver. 22.08.2020).
Morse, N. (2020). The Museum as a Space of Social Care. Routledge.

Museo Archeologico Nazionale di Venezia (2020). \#ungiocodaragazzi. https://www.facebook.com/archeovenezia/

Museo Benozzo Gozzoli (2020). Materiali e attività. http://www.museobenozzogozzoli.it/it_IT/museichiusimuseiaperti.html?inheritRedirect $=$ true

Museo d'Arte Sorlini (2020). Discovery Museum Challenge. https://www.museomartes.com/discoverymuseum-challenge/ (ver. 22.08.2020).

Museo dei Bambini di Milano (2020). MUBA legge CORRAINI. https://www.facebook.com/watch/?v=547674 445852903\&extid=dzHxkwjBly7nPyyA

Museo Nazionale della Scienza e della Tecnologia Leonardo da Vinci (2020). Colle rinascimentali \#storieaportechiuse. https://www.youtube.com/ watch?v=Q1ICyQ5Ic-I

Museo Nazionale delle Arti del XXI secolo (2020). \#iorestoacasa con il MAXXI. https://www.maxxi.art/ programmi-educativi/iorestoacasa-con-il-maxxi/

Nardi, E. (2011). Forme e messaggi del museo. FrancoAngeli.

NEMO (2020). Survey on the impact of the COVID-19 situation on museums in Europe. https://www.ne-mo. org/fileadmin/Dateien/public/NEMO_documents/ NEMO_Corona_Survey_Results_6_4_20.pdf

Nofal, E., Reffat, M., \& Vande Moere, A. (2017). Phygital heritage: an approach for heritage communication. In D. Beck, C. Allison, L. Morgado, J. Pirker, F. Khosmood, J. Richter \& C. Gütl (eds.) (2017) Workshop, Long and Short Paper, and Poster Proceedings from the Third Immersive Learning Research Network Conference (pp. 220-229). iLRN.

Nuzzaci, A. (2012). La didattica museale tra pedagogical literacy, heritage literacy e multiliteracies: Costruire il profilo del letterato del 21 secolo. Pensa MultiMedia.

Panciroli, C. (2014). Un'esperienza al patrimonio è educativa quando...: progetti, linguaggi e strumenti a confronto. In C. Panciroli \& F. D. Pizzigoni (eds.) (2014). Il museo come officina di esperienze con il patrimonio: l'esempio del MOdE (pp. 19-27). QuiEdit.

Panciroli, C. (2019). Documentare per creare nuovi significati: i musei virtuali. In P.C. Rivoltella \& P. G. Rossi (eds.) (2019), Tecnologie per l'educazione (83-94). Pearson Italia.

Poce, A. (2017). Il patrimonio culturale per lo sviluppo delle competenze nella scuola primaria. FrancoAngeli.

Pomian, K. (1978). Collezione in Enciclopedia Einaudi, 3. Einaudi.

Rivoltella, P. C. (2015). Le virtù del digitale: Per unetica dei media. Morcelliana. 
Steier, R., Pierroux, P., \& Krange, I. (2015). Embodied interpretation: Gesture, social interaction, and meaning making in a national art museum. Learning, Culture and Social Interaction, 7, 28-42. https://doi. org/10.1016/j.lcsi.2015.05.002

Stelarc (1994). Da strategie psicologiche a cyberstrategie: prostetica, robotica ed esistenza remota in P. L. Capucci (ed.) (1994), Il corpo tecnologico: l'influenza delle tecnologie sul corpo e sulle sue facoltà (pp. 61-76). Baskerville.

United Nations (1989). Convention on the Rights of the Child. https://www.ohchr.org/Documents/ProfessionalInterest/crc.pdf 